\title{
Clostridial infection in children
}

\author{
I. BROOK
}

Department of Pediatrics, Georgetown University School of Medicine, Washington DC, USA

\begin{abstract}
Summary. A survey of the isolation of Clostridium spp. from 1543 specimens sent to anaerobic microbiology laboratories revealed 113 isolates from 107 specimens ( $7.0 \%$ of all specimens) from 96 children. The isolates comprised 43 (38\%) unidentified Clostridium spp., 37 (33\%) C. perfringens, 13 (12\%) C. ramosum, five (4\%) C. innocuum, six (5\%) C. botulinum, three (3\%) C. difficile, two (2\%) C. butyricum, and one isolate each of C. bifermentans, $C$. clostridiiforme, $C$. limosum and $C$. paraputrificum. Most clostridial isolates were from abscesses (38), peritonitis (26), bacteraemia (10), and chronic otitis media (7). Predisposing or underlying conditions were present in $31(32 \%)$ cases. These were immunodeficiency (12), malignancy (9), diabetes (7), trauma (7), presence of a foreign body (6) and previous surgery (6). The clostridia were the only bacterial isolates in $14(15 \%)$ cases; $82(85 \%)$ cases had mixed infection. The species most commonly isolated with clostridia were anaerobic cocci (57), Bacteroides spp. (B. fragilis group) (50), Escherichia coli (22), pigmented Prevotella or Porphyromonas spp. (18) and Fusobacterium spp. (10). Most Bacteroides and Escherichia coli isolates with clostridia were from abdominal infections and skin and soft tissue infections adjacent to the rectal area; most pigmented Prevotella and Porphyromonas isolates were from oropharyngeal, pulmonary, and head and neck sites. Antimicrobial therapy was given to all patients, in conjunction with surgical drainage in $34(35 \%)$. Only two patients died. These data illustrate the importance of Clostridium spp. in paediatric infections.
\end{abstract}

\section{Introduction}

Recognition of the role of anaerobic bacteria in paediatric infections has increased recently. ${ }^{1}$ However, most of the interest has focused on Bacteroides spp.; other groups of organisms such as Clostridium spp. have not been studied as adequately. This article summarises experience of the isolation of clostridial species from infections in children over a period of 17 years. Some of the data were published previously in separate articles describing the role of anaerobic bacteria in paediatric infections, ${ }^{1}$ but the role of the clostridia was not highlighted in those studies, as they were isolated from only a small proportion $(<10 \%)$ of the patients. This review also includes cases not previously presented that were seen after those studies were completed.

\section{Patients and methods}

\section{Patients}

The children included in the study were investigated between June 1973 and June 1990 at the following hospitals: University of California Medical Center, Los Angeles County Medical Center and Serra Memorial Hospital in Los Angeles, CA; Children's Hospital National Medical Center and South-East Medical Center in Washington, DC; and National Naval Medical Center in Bethesda, MD. Records from the clinical microbiology laboratories were reviewed to identify patients with clostridial infections. The case histories of all patients from whom Clostridium spp. had been isolated were reviewed to ascertain the presence and site of infection, associated microorganisms, disease process, and possible predisposing or associated conditions.

\section{Microbiological investigations}

Blood was collected aseptically from patients suspected of having bacteraemia and was inoculated $(10 \% \mathrm{v} / \mathrm{v})$ into one bottle each of two commercially 
obtained broth media, both under vacuum and with $\mathrm{CO}_{2}$. For other sites, the microbiology laboratories accepted only specimens that were collected properly by a method that avoided contamination by the normal skin or mucosal flora, ${ }^{2}$ and submitted in transport media appropriate for anaerobic bacteria. These were specimens obtained during surgery, aseptic aspiration of body fluids, or needle or biopsy aspiration of abscesses or body cavities. Aspirates of lungs were obtained through transtracheal aspiration or biopsy. Urine was collected through suprapubic aspiration. When possible, pus and fluid samples were collected and transported to the laboratory in syringes within $30 \mathrm{~min}$. Most specimens, except blood, were submitted in Port-A-Cul transport swab or liquid systems (BBL, Cockeysville, MD, USA). However, exact records of the transport media used were not available in all instances.

The specimens were inoculated on to pre-reduced vitamin $\mathrm{K}_{1}$-enriched Brucella blood agar, an anaerobic blood agar plate containing kanamycin and vancomycin, an anaerobic blood agar plate containing colistin sulphate and naladixic acid, and an enriched thioglycolate broth (containing haemin, sodium carbonate and vitamin $\left.\mathrm{K}_{1}\right){ }^{2}$ Cultures were incubated in GasPak jars (BBL) and examined after 48 and $96 \mathrm{~h}$. Plates that showed growth were held until the organisms were processed and identified. All cultures that showed no growth were incubated for at least 5 days. Organisms were identified by the API Anaerobic System (Analytab Products, Plainview, NY, USA) or the Minitek System (BBL). When complete identification was not possible by these methods, other carbohydrate tests (Scott Laboratories, Fiskeville, RI, USA) and gas-liquid chromatography ${ }^{2,3}$ were performed as needed to identify the organisms. The criteria for identification were according to previously published guidelines. ${ }^{2,3}$

\section{Results}

From the 1543 specimens examined for anaerobic bacteria, 113 clostridia were isolated from 107 specimens $(7.0 \%$ of all specimens examined) from 96 patients. The isolates comprised $43(38 \%)$ unidentified Clostridium spp., 37 (33\%) C. perfringens, 13 (12\%)C. ramosum, five $(4 \%)$ C. innocuum, six $(5 \%)$ C. botulinum, three $(3 \%)$ C. difficile, two $(2 \%)$ C. butyricum and one isolate each $(1 \%)$ of $C$. bifermentans, $C$. clostridiiforme, C. limosum and C. paraputrificum (tables I and II). Clinical records were not available for another six isolates from five patients. These cases were not included in the study.

Most infections were polymicrobial-in $82(85 \%)$ patients-but in $14(15 \%)$ patients a Clostridium sp. was isolated in pure culture. Most clostridia were isolated from abscesses ( 38 isolates; $34 \%$ ), peritonitis $(26 ; 23 \%)$, bacteraemia $(10 ; 9 \%)$, including one neonatal bacteraemia, and chronic otitis media

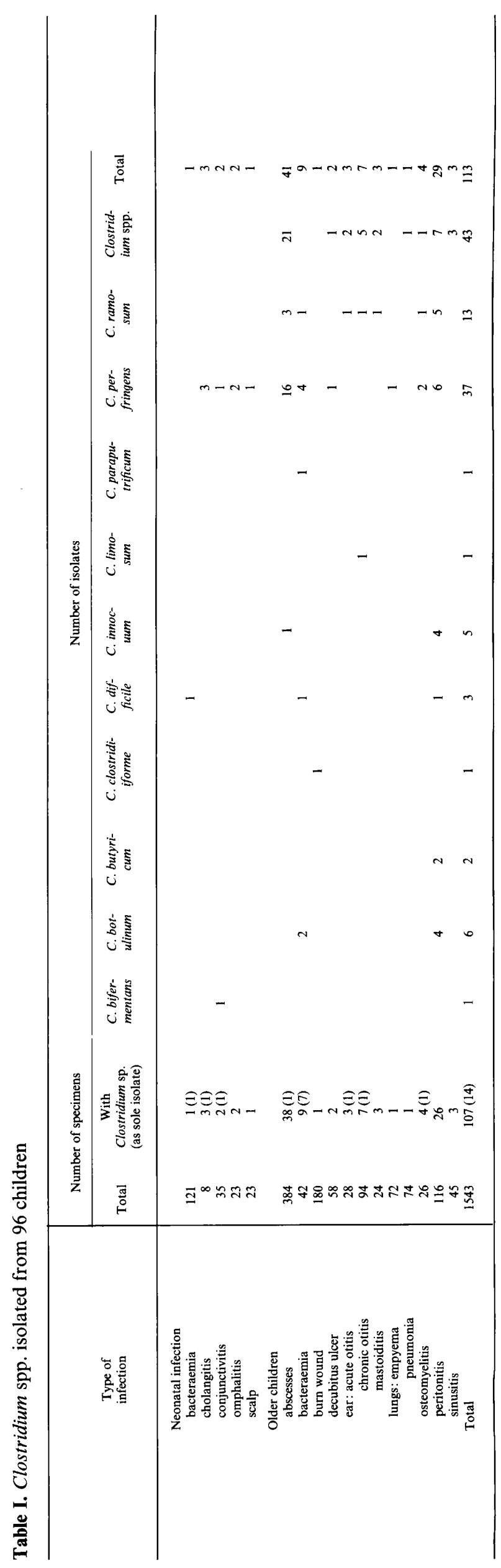


Table II. Clostridium sp. isolated from 38 abscesses in children

\begin{tabular}{|c|c|c|c|c|c|c|}
\hline \multirow{2}{*}{ Type of abscess } & \multirow{2}{*}{$\begin{array}{c}\text { Total } \\
\text { number of } \\
\text { abscesses }\end{array}$} & \multirow{2}{*}{$\begin{array}{c}\text { Number of } \\
\text { specimens with } \\
\text { Clostridium } \mathrm{sp} \text {. }\end{array}$} & \multicolumn{4}{|c|}{ Number of isolates of } \\
\hline & & & C. innocuum & C. ramosum & C. perfringens & Clostridium sp. \\
\hline Abdominal & 38 & 12 & 1 & 2 & 5 & 7 \\
\hline Intracranial & 40 & 1 & & & 1 & \\
\hline Liver & 8 & 1 & & & 1 & \\
\hline Perinephric & 6 & 1 & & & 1 & \\
\hline Perirectal & 28 & 3 & & & 2 & \\
\hline Peritonsillar & 16 & 3 & & & & 3 \\
\hline Pilonidal & 25 & 4 & & & 2 & 2 \\
\hline Retropharyngeal & 14 & 2 & & & & 2 \\
\hline Skin and soft tissue & 195 & 6 & & 1 & 2 & 3 \\
\hline Subphrenic & 14 & 5 & & & 2 & 4 \\
\hline Total & 384 & 38 & 1 & 3 & 16 & 21 \\
\hline
\end{tabular}

$(7 ; 6 \%)$. Predisposing or underlying conditions were noted in $31(32 \%)$ cases. A single condition was noted in $18(19 \%)$ instances, two such conditions in 10 $(10 \%)$ and three conditions in three $(3 \%)$. These were immunodeficiency $(12 ; 13 \%)$, malignancy $(9 ; 10 \%)$, diabetes $(7 ; 8 \%)$, trauma $(7 ; 8 \%)$, presence of a foreign body $(6 ; 6 \%)$ and previous surgery $(6 ; 6 \%)$.

There were 240 other isolates mixed with Clostridium spp. in the 93 specimens from mixed infections; $168(70 \%)$ were anaerobes and $72(30 \%)$ were aerobes or facultative species. The number of isolates varied from two to seven (average 2.6/specimen, 1.8 anaerobic and 0.8 aerobic or facultative). The organisms isolated most commonly with clostridia were anaerobic cocci (57), Bacteroides spp. (50), pigmented Prevotella and Porphyromonas spp. (18), Fusobacterium spp. (10), Escherichia coli (22), non-haemolytic streptococci (10) and $\alpha$-haemolytic streptococci (7). Most Bacteroides and E. coli isolates were cultured with clostridia from intra-abdominal and peri-rectal skin and soft tissue infections, whereas most pigmented Prevotella and Prophyromonas isolates were from oropharyngeal, pulmonary and head and neck sites.

Of the 79 patients with mixed infection, six were neonates (table I). One had conjunctivitis in which $C$. bifermentans was mixed with a Peptostreptococcus $\mathrm{sp}$. Similar organisms were also isolated from the mother's cervix immediately after delivery. The patient responded to therapy with penicillin eye drops. Two neonates had omphalitis; one was born to a mother with premature rupture of membranes and amnionitis. Two had ascending cholangitis following hepatic portoenterostomy for biliary atresia, and one had a scalp abscess as a result of fetal monitoring; the latter patient also had bacteraemia due to $B$. fragilis.

Of the 73 other patients with mixed infections, 37 had abscesses (table II), of which the most common were abdominal (12), rectal area (7), skin and soft tissue (6), subphrenic (5), peritonsillar (3) and retropharyngeal (2). The other organisms isolated most commonly with clostridia from abscesses were Peptostreptococcus spp. (25 isolates), Bacteroides spp. (19),
E. coli (17), pigmented Prevotella and Porphyromonas spp. (7) and Staphylococcus aureus (5). Peptostreptococci were isolated mostly from abdominal, orofacial, and head and neck abscesses; $B$. fragilis and $E$. coli were isolated predominantly from abdominal and peri-rectal abscesses.

Amongst the patients with mixed infection of the ear (table I), two had acute and six had chronic otitis media and three had chronic mastoiditis. The most common organisms accompanying the clostridia were Peptostreptococcus spp., Bacteroides spp. and gramnegative aerobic or facultative bacteria. Cholesteretoma was present in three of the patients with chronic otitis media and in two with chronic mastoiditis. The three clostridial isolates from chronic sinusitis were mixed with oral organisms. There was only a single Clostridium isolate ( $C$. clostridiiforme) from 180 patients with burn wounds; it was isolated in mixed culture with $B$. fragilis from a burn of the thigh. In specimens from the respiratory tract, the single clostridial isolate from aspiration pneumonia was mixed with Klebsiella pneumoniae in a child who suffered from mental retardation and the one patient with a clostridial isolate from empyema had an associated lung abscess (which was not cultured). There were 26 clostridial isolates from cases of peritonitis; 19 were from children with ruptured appendix, five with abdominal trauma, and two with spontaneous peritonitis. The clostridia were mostly mixed with Peptostreptococcus spp., Bacteroides spp. and enterobacteria. Of the three osteomyelitis isolates, two were from temporal bone infections, one associated with chronic otitis media and one with chronic mastoiditis, and one was from an infected coccyx associated with a decubitus ulcer. The two isolates from this and another decubitus ulcer were from the buttocks area. Organisms of intestinal origin were mixed with these clostridia. There were two cases of polymicrobial bacteraemia, one in a 5-year-old child with a rectal abscess and leukaemia, and the other with abdominal trauma and ruptured viscus.

Clinical and microbiological data on the 14 patients from whom clostridia were isolated in pure culture are 
Table III. Summary of 14 patients infected with a Clostridium sp. as sole isolate

\begin{tabular}{|c|c|c|c|c|c|c|c|}
\hline $\begin{array}{l}\text { Case } \\
\text { no. }\end{array}$ & Age & Sex & Diagnosis & Culture site & $\begin{array}{c}\text { Clostridium } \\
\text { sp. } \\
\text { isolated }\end{array}$ & Therapy & Outcome \\
\hline I & $2 \mathrm{~d}$ & $\mathrm{~F}$ & Conjunctivitis & Conjunctival sac & C. perfringens & $\begin{array}{l}\text { Penicillin } 2 \% \\
\text { eye drops }\end{array}$ & Cured \\
\hline 2 & $3 \mathrm{~m}$ & $\mathbf{M}$ & $\begin{array}{l}\text { Cholangitis; following } \\
\text { porto-enterostomy }\end{array}$ & Bile duct & C. perfringens & $\begin{array}{l}\text { Penicillin }+ \\
\text { gentamicin }\end{array}$ & Cured \\
\hline 3 & $1 \mathrm{~m}$ & $\mathbf{M}$ & Necrotising enterocolitis & Blood, peritoneal cavity & C. difficle & $\begin{array}{l}\text { Penicillin } \\
\quad+\text { gentamicin }\end{array}$ & Died \\
\hline 4 & 1 y $4 \mathrm{~m}$ & $\mathbf{M}$ & $\begin{array}{l}\text { Bacteraemia: necrotising } \\
\text { enterocolitis }\end{array}$ & Blood, peritoneal cavity & C. difficile & $\begin{array}{l}\text { Ampicillin } \\
\text { + gentamicin }\end{array}$ & Died \\
\hline 5 & $10 y$ & F & Sepsis; sickle cell disease & Blood & C. paraputrificum & Penicillin & Cured \\
\hline 6 & $13 \mathrm{y}$ & $\mathbf{M}$ & Sepsis; sickle cell disease & Blood, peritoneal cavity & C. perfringens & $\begin{array}{l}\text { Penicillin } \\
\quad+\text { gentamicin }\end{array}$ & Cured \\
\hline 7 & 3 y $6 \mathrm{~m}$ & $F$ & Sepsis; perforated viscus & Blood & C. ramosum & Penicillin & Cured \\
\hline 8 & 7 y $6 \mathrm{~m}$ & $\mathbf{M}$ & Sepsis; diarrhoea & Blood & C. perfringens & Penicillin & Cured \\
\hline 9 & $15 y$ & $\mathrm{~F}$ & Sepsis & Blood & C. perfringens & Penicillin & Cured \\
\hline 10 & $10 \mathrm{y}$ & $\mathrm{F}$ & Sepsis; leukaemia & Blood & C. perfringens & Penicillin & Died \\
\hline 11 & $13 \mathrm{y}$ & $\mathbf{M}$ & Post-traumatic osteomyelitis & Left femur & C. perfringens & Penicillin & Cured \\
\hline 12 & $11 \mathrm{y}$ & $\mathrm{F}$ & Rectal abscess; leukaemia & Abscess cavity & C. perfringens & Amoxycillin & Cured \\
\hline 13 & $3 y$ & $\mathbf{M}$ & Chronic otitis media & Middle ear & C. limosum & Amoxycillin & Cured \\
\hline 14 & 3 y $6 \mathrm{~m}$ & $\mathbf{M}$ & Acute otitis media & Middle ear & C. ramosum & Amoxycillin & Cured \\
\hline
\end{tabular}

listed in table III. These included eight children with sepsis or bacteraemia (including one neonate) (patients nos. 3-10), two with otitis (one acute and one chronic) and one case each of neonatal conjunctivitis, cholangitis, osteomyelitis, and peri-rectal abscess. Two patients with $C$. perfringens infection died $(2 \%)$; one was a neonate (patient no. 4) with necrotising enterocolitis, and the other (no. 10) had lymphocytic leukaemia.

Antimicrobial therapy was administered to all patients and combined with surgical drainage or correction of pathological abnormalities in $34(35 \%)$. The antimicrobial agents administered were penicillin in 43 , an aminoglycoside in 31 , clindamycin in 28 , a cephalosporin in 19 , metronidazole in 15 , vancomycin in five and erythromycin in two.

\section{Discussion}

This review confirms that clostridia are commonly isolated from various infections in children. They are especially prevalent in abscesses (mostly abdominal, rectal area and oropharyngeal), peritonitis and otolaryngological infections, as has been shown in adults. ${ }^{4}$ The distribution of clostridia in these infections is explained by their prevalence in the normal gastrointestinal and cervical flora from where they may originate..$^{5}$

The clostridia found most frequently in clinical infections are $C$. perfringens, $C$. ramosum, C. botulinum and $C$. innocuum. ${ }^{4} C$. perfringens is a member of the intestinal flora of man and animals, is found in soil and is the most frequently encountered histotoxic clostridial species. It elaborates several necrotising extracellular toxins and is easily isolated and identified in the clinical laboratory. C. perfringens seldom produces spores in vivo. It can be recognised in direct smears of a purulent exudate by the presence of stout gramvariable rods of varying length, frequently surrounded by a capsule, and can cause a devastating illness with high mortality. ${ }^{1,4}$ Clostridial bacteraemia is associated with extensive tissue necrosis, haemolytic anaemia and renal failure. ${ }^{4} C$. perfringens accounted for $48 \%$ of all clostridial isolates in adults and children in a recent survey in two military hospitals, ${ }^{6}$ and was isolated primarily from wounds ( $26 \%$ of isolates), blood $(16 \%)$, abdomen $(14 \%)$, and obstetrical and gynaecological infections $(13 \%)$. C. butyricum is isolated mostly from infections of the abdomen, abscesses, bile, wounds and blood. ${ }^{1,4}$ It was reported ${ }^{7}$ from blood cultures from 12 neonates with necrotising enterocolitis in one study, but the association of this species with the disease has not been established. Strains of $C$. perfringens, $C$. butyricum and $C$. difficile have been isolated from blood and peritoneal cultures of infants with necrotising enterocolitis and with sudden death syndrome. ${ }^{8}$ Clostridium spp. have also been isolated from children with bacteraemia of gastrointestinal origin $^{1}$ and with sickle cell disease. ${ }^{9} C$. difficile is the causative agent of antibiotic-associated and spontaneous diarrhoea and pseudomembranous colitis in adults. ${ }^{10,11}$ Infants are not susceptible to intestinal infection with $C$. difficile, but there were three isolates from bacteraemia or peritonitis in this study and its isolation has been reported in children from infections of the peritoneal cavity, blood and lungs, and from wounds and central nervous system infections. ${ }^{1}$

Clostridia can act synergically with other aerobic and anaerobic bacteria. ${ }^{12}$ As a result, polymicrobial infections that involve clostridia may be more destructive and more difficult to eradicate. The synergy between the different bacterial strains may be due to inhibition of phagocytosis and intracellular killing, ${ }^{13}$ production of essential growth factors, ${ }^{14}$ or lowering of redox potentials in host tissues. ${ }^{15}$ 
C. botulinum is usually associated with food poisoning $;^{1,4}$ botulism is an intoxication caused by ingestion of contaminated food containing its highly potent neurotoxin. However, wound infections caused by proteolytic strains of types A and B have been reported with increasing frequency and can also produce botulism. This was not observed in the present study.

The management of clostridial infections comprises a combination of antimicrobial therapy and surgical intervention. Drainage of pus and debridement of necrotic tissues are integral to therapy. Most infections from which clostridia are isolated require antimicrobial therapy. Clostridia are generally susceptible to most antibiotics used for the treatment of anaerobic infections, ${ }^{16}$ including $\beta$-lactam antibiotics, macrolides, carbapenems, imidazoles and chloramphenicol. Susceptibility to antimicrobial agents was not recorded in the present study, but general guidelines can be based upon previously reported susceptibility data.

Increased resistance of clostridia to several anti-

\section{References}

1. Brook I. Pediatric anaerobic infections: diagnosis and management. A textbook. Philadelphia, PA, Mosby Publisher, Inc. 1989.

2. Sutter VL, Citron DM, Edelstein MAC, Finegold SM. Wadsworth bacteriology manual, 4th edn. Belmont, CA, Star Publishing Co. 1985.

3. Holdeman LV, Cato EP, Moore WEC. Anaerobe laboratory manual, 4th edn. Blacksburg, VA, Virginia Polytechnic Institute and State University. 1977.

4. Finegold SM. Anaerobic bacteria in human disease. New York, Academic Press. 1977.

5. Rosebury T. Microorganisms indigenous to man. New York, McGraw-Hill Book Co. 1966.

6. Brook I. Recovery of anaerobic bacteria from clinical specimens in 12 years at two military hospitals. J Clin Microbiol 1988; 26: 1181-1188.

7. Howard MF, Flynn DM, Bradley JM, Noone P, Szawatkowski M. Outbreak of necrotising enterocolitis caused by Clostridium butyricum. Lancet 1977; 2 : 1099-1102.

8. Cashore WJ, Peter G, Lauermann M, Stonestreet BS, Oh W. Clostridia colonization and clostridial toxin in neonatal necrotizing enterocolitis. J Pediatr 1981; 98: 308-311.

9. Brook I, Gluck RS. Clostridium paraputrificum sepsis in sickle cell anaemia. South Med J 1980; 73: 1644-1645.

10. Viscidi RP, Bartlett JG. Antibiotic-associated pseudomembraneous colitis in children. Pediatrics $1981 ; 67$ : 381-386.

11. Brook I. Isolation of toxin producing Clostridium difficile from two children with oxacillin- and dicloxacillin-associated diarrhea. Pediatrics 1980; 65: 1154-1156. microbial agents has been reported by others. ${ }^{17-20} \mathrm{~A}$ survey of isolates ${ }^{17}$ from the Mayo Clinic described multi-resistant Clostridium spp. Isolates of C. clostridiiforme, $C$. innocuum and $C$. ramosum were resistant to achievable serum concentration "breakpoints" of chloramphenicol (10-33\%), clindamycin (23-30\%), penicillin (16-26\%), metronidazole $(11-12 \%)$ and cefoxitin $(22-48 \%)$. Isolates of $C$. butyricum that produced $\beta$-lactamase and were resistant to penicillins have been described. ${ }^{17,18}$ These investigators also noted that a minority of $C$. perfringens strains were resistant to clindamycin $(14 \%)$ and penicillin $(5 \%)$. Marrie et al. ${ }^{20}$ also reported increased resistance to penicillin in $C$. perfringens isolates in Canada; $50 \%$ of isolates were inhibited by $0.25 \mathrm{mg} / \mathrm{L}$ but the MIC 90 was $8 \mathrm{mg} / \mathrm{L}$. The susceptibility of Clostridium spp. needs to be monitored constantly to ensure correct empiric therapy.

I gratefully acknowledge the technical support of L. Calhoun, P. Yocum, J. Perry and E. H. Frazier, and the editorial assistance of Sarah Blaisdell.

12. Brook I, Hunter V, Walker RI. Synergistic effect of Bacteroides, Clostridium, Fusobacterium, anaerobic cocci, and aerobic bacteria on mortality and induction of subcutaneous abscesses in mice. $J$ Infect Dis 1984; 149: 924-928.

13. Ingham HR, Tharagonnet D, Sisson PR, Selkon JB, Codd AA. Inhibition of phagocytosis in vitro by obligate anaerobes. Lancet $1977 ; 2$ : 1252-1254.

14. Lev M, Keudell KC, Milford AF. Succinate as a growth factor for Bacteroides melaninogenicus. J Bacteriol 1971; 108: 175-178.

15. Mergenhagen SE, Thonard JC, Scherp HW. Studies on synergistic infections. I. Experimental infections with anaerobic streptococci. $J$ Infect Dis $1958 ; 103$ : 33-44.

16. Sutter VL, Finegold SM. Susceptibility of anaerobic bacteria to 23 antimicrobial agents. Antimicrob Agents Chemother 1976; 10: 736-752.

17. Edson RS, Rosenblatt JE, Lee DT, McVey EA. Recent experience with antimicrobial susceptibility of anaerobic bacteria: increasing resistance to penicillin. Mayo Clin Proc 1982; 57: 737-741.

18. Carlson JR, Sherrill JM, Rosenblatt JE, McCarthy LR. Penicillinase activity in three strains of Clostridium butyricum. Curr Microbiol 1981; 5: 251-254.

19. Brazier JS, Levett PN, Stannard AJ, Phillips KD, Willis AT. Antibiotic susceptibility of clinical isolates of clostridia. $J$ Antimicrob Chemother 1985; 15: 181-185.

20. Marrie TJ, Haldane EV, Swantee CA, Kerr EA. Susceptibility of anaerobic bacteria to nine antimicrobial agents and demonstration of decreased susceptibility of Clostridium perfringens to penicillin. Antimicrob Agents Chemother $1981 ; 19: 51-55$. 